\title{
Network Pharmacology-based on the Active Components and Molecular Mechanisms of Andrographis paniculata (Burm. f.) Wall. ex Nees in Treating Inflammation
}

\author{
Wen-ping Xiao ${ }^{1,2, *}$, Wan-ju Zhang ${ }^{1}, X$-jie Xiong ${ }^{1}$, Lan-ying Xu' ${ }^{1}$ \\ ${ }^{1}$ Hubei Key Laboratory of Processing and Application of Catalytic Materials, College of Chemistry and Chemical Engineering, \\ Huanggang Normal University, Hubei Province, CHINA. \\ ${ }^{2}$ Faculty of Pharmacy, Hubei University of Chinese Medicine, Wuhan, CHINA.
}

\begin{abstract}
Background: Andrographis paniculata (Burm. f.) Wall. ex Nees (AP) is a medicinal plant traditionally used as anti-inflammation and anti-bacteria. The role of AP in inflammation has been evaluated in several studies. But, the exact mechanism is unclear. In the present study, network pharmacology was used to explore the anti-inflammation constituents of AP and its anti-inflammation mechanism. Materials and Methods: The chemical components of AP. were screened by TCMSP database, in combination with literature. The effective compounds were filtrated by drug likeness and pharmacokinetic characteristics (ADMET). The target genes of effective compounds were predicted by Swisspredict database. The anti-inflammation genes were found in databases, and antiinflammation related target genes were screened by comparison. The functions of target genes and related pathways were analyzed and screened by database, the ComponentTarget-Pathways network of anti-inflammation effect of AP was established by using Cytoscape software. AutoDock was used to verify the molecular docking between the molecule and the target. Results: The research showed that the anti-inflammatory effective ingredients in AP were moslosooflavone, rographidine F_qt, rographin, 14-deoxy11-oxo-andrographolide, mono-O-methylwightin. The mechanisms were involved in PI3kAkt signaling pathway, Neuroactive ligand-receptor interaction, Measles and so on. The top 3 targets were PIK3CG, PIK3CA and PIK3CD and they were all enriched in PI3kAkt signaling pathway. Molecular docking showed that the five components had high binding activity with the screened target proteins, which provided further verification for subsequent network analysis. Conclusion: The anti-inflammatory activity mechanism of AP was characterized by multiple components, multiple targets and multiple pathways. The results of network pharmacology can provide certain scientific basis for subsequent experimental research.
\end{abstract}

Key words: Andrographis paniculata (Burm.f.) Wall. ex Nees, Network pharmacology, Inflammation, Docking.

\section{INTRODUCTION}

Andrographis paniculata (Burm. f.) Wall. ex Nees (AP) is an important medicinal plant and widely used around the world. ${ }^{1}$ It has been used as an anti-inflammator herb medicine in China for many years. ${ }^{2}$ With increasing interest in natural products, novel pharmacology effects of AP have been expanded. ${ }^{3}$
The chemical components of AP are complex include diterpenoids, flavonoids and polyphenols. Among the single compounds extracted from AP rographolide is the major one in terms of bioactive properties and abundance. ${ }^{4}$ It has been demonstrated that andrographolide and its derivatives has anti-inflammatory effects by
Submission Date: 07-10-2020; Revision Date: 18-12-2020; Accepted Date: 15-05-2021

DOI: 10.5530/ijper.55.3.149 Correspondence: Dr. Wen-ping Xiao College of Chemistry and Chemical Engineering, Huanggang Normal University, Hubei Province, CHINA.

Phone: +86-0173-8833611 Email id: 593223995@qq.com

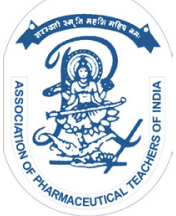

www.ijper.org 
decreasing serum iNOS activity, NO production, PEG2..$^{5}$ 14-deoxyandrographolide shows anti-inflammatory activity by docking. ${ }^{6}$ AP show potent anti-inflammatory effect on pathogen-induced PID in rats, with a potential mechanism of inhibiting the NF- $x \mathrm{~B}$ signal pathway. ${ }^{7}$ The anti-inflammatory mechanism of AP has been studied by some scholars, ${ }^{5}$ but their research was lack of systematic the active components and targets were unclear. The active compounds have been identified by pharmacokinetic research, ${ }^{8,9}$ but the signal pathway researches were missing. So the present research studies anti-inflammatory constituents of AP and its antiinflammatory mechanisms by network pharmacology.

Hopkins introduced network pharmacology in 2008 by using visual web software and a series of algorithms combined with comprehensive analysis of existing relevant databases. ${ }^{10}$ Network pharmacology is a new subject of establishing a multilevel network of diseasegene-drug; drug targets can be predicted by the network in the discovery of new drugs. ${ }^{11}$

In present research, we used network pharmacology to study the anti-inflammatory compounds of AP and its anti-inflammatory mechanism. The anti-inflammatory compounds of AP were filtrated by TCMSP database, in combination with literature. The anti-inflammatory targets of the compounds were screened by Swisspredict Swiss predict and Gene Cards databases. The protein interaction network Figure was plotted by String database and Cytoscape 3.6.1 software. The anti-inflammatory targets of AP were imported into David 6.8 database andthe results were analyzed by the Gene Ontology (GO) and KEGG pathway. Cytoscape 3.6.1 software was used to draw the Component-Target-Pathway interaction network. The molecular docking between components and targets were accomplished by Autodock. At last, an interaction network of the component-target-pathway of AP was established, the functions and signal channels were analyzed for subsequent exploring the mechanisms of AP in treating anti-inflammation.

\section{MATERIALS AND METHODS}

\section{Active compounds screening}

The chemical compounds of AP were screened from the Traditional Chinese Medicine Systems (http://lsp. nwu.edu.cn/tcmsp.php)database, in combination with literature. The chemical structures formats were found from Pubchem (https://pubchem.ncbi.nlm.nih.gov/). If the specific structures could not be found in the databases, the original research literature was searched, the structure was drawn by Chem Bio Draw 16.0 software, the files were saved in mol2 or sdf formats. Many of the traditional Chinese medicine are oral dosage, which need to undergo absorption, distribution, metabolism, excretion processes $\mathrm{ADME}^{12}$ to reach the target positions. To filtrate potential effective components, a comprehensive model including oral bioavailability (OB) and drug likeness (DL) was used to predict the ADME properties of the components. Considering the low $\mathrm{OB}$ of the active components, $\mathrm{OB} \geq 30 \%$ was used as the standard in the present research. The component $\mathrm{DL} \geq 0.18$ was considered to have a certain similarity with the drug in the DrugBank database. The components filtrated by $\mathrm{OB}$ and DL were used as the effective components of AP.

\section{Compound Target Screening}

Drug molecules develop their active ability by binding to target proteins. The mechanism of a drug's, the interactions drug-target proteins must be established. The molecular structures in mol 2 or sdf formats, which were screened out in 2.1, were uploaded on Swiss Target Prediction database (http://www.swisstargetprediction. ch/index.php). The UniProt column of the downloaded table represented the UniProt Knowledgebase of the potential protein targets that can bind to active compounds. The proteins were transformed into the official symbol format of the gene targets by using the Retrieve/ID mapping tool in UniProt (http://www. uniprot.org/), and the potential gene targets were saved. Collection of the Anti-inflammatory Targets

By searching for the keyword "inflammation" on GeneCards and MalaCards, the inflammation-related genes were obtained and compared with the potential target genes of the active compounds. The potential target genes of the active compounds of AP for antiinflammation were obtained.

\section{Construction and Analysis of the Protein-Protein Interaction Network}

To explore the functions of the target proteins in antiinflammatory mechanism, the potential inflammatory related target proteins of AP obtained from 2.3 were uploaded on String10.5 online software (http:// string-db.org), revised the "species" as "humans," and the Protein-Protein Interaction network Figure was obtained. Protein-Protein interactions including direct and indirect interactions between proteins that were predicted by String and the scores to each protein interaction information were evaluated and calculated. When the score was higher, the degree of proteins interaction was higher. The node A, node B, Combined 
score were exported from the String database, the results were imported to Cytoscape 3.6.1 software for visual analysis, so the network analysis was obtained. The node size was set, color, degree value, size, edge thickness reflected combined score. The ProteinProtein Interaction network was established.

\section{Target pathway annotation and analysis}

The anti-inflammatory targets of AP screened from 2.3 were imported to David 6.8 (https://David.ncifcrf. gov) in the format of Gene Symbol. The identifier was set as Official Gene Symbol, List Type was set as Gene List to restrict the research in human species. GO and KEGG pathway analysis were performed on the antiinflammatory targets of AP, the results were downloaded and saved. When the difference between pathways was significant, $P<0.05$. The data were classified according to the size of $P$-value, the pathways were ranked from high to low. The Figure was plotted by GraphPad Prism 5.0 software.

The Kyoto Encyclopedia of Genes and Genomes (KEGG, https://www.kegg.jp/) was established by the Kanehisa Laboratory of the Bioinformatics Center of Kyoto University, Japan in 1995. The signaling pathways and target genes were analyzed by the network pharmacology. KEEG also can be use to understand the signal pathways involved in target genes. The GO (Gene Ontology) database standardizes gene products from functions, participating biological pathways and cell localization. It covers three aspects of biology, namely Cell Component (CC), Molecular Function (MF), Biological Process (BP).

\section{Component-Target-Pathway Network Construction}

An active component-target-pathway network was built by Cytoscape 3.6.1 software. The active components, target genes, enriched pathways were imported to Cytoscape 3.6.1 software as the node. If there was a connection between two nodes, the connection was shown by edge. The two groups of networks were merged by the merge function. Finally, the ComponentTarget-Pathway network was established, in which high degree compounds were regarded as important active compounds.

\section{Molecular Docking}

High degree target genes in 2.4 and 2.6 were analyzed. According to the results of KEGG enrichment, the pathways with higher targets numbers were selected to key targets. At the same time, the genes suitable for the analysis of the targets were obtained through comparative analysis from the literature and database. In 2.6, the top 5-degree ranking compounds were selected.
The protein structure and active components were modified by Pymol software and AutoDockTools 1.5.6. The addition of hydrogen atoms, merging nonpolar hydrogen atoms, rotatable torsion bond were done by Pymol software, followed by gridbox construction of each target protein. The molecular docking experiments of the processed protein and effective compounds were finished by AutoDockTools 1.5.6, the binding energy was calculated by the Lamarckian genetic algorithm. Molecular docking experiments were finished under the same conditions, the obtained binding energy was used as a standard to filtrate the effective compounds.

\section{RESULTS}

\section{Active component screening}

According to the literature data, the functional components of AP were flavonoids and terpenoids, which were also the main functional compounds. Based on literature data, $\mathrm{OB} \geq 30 \%$ and $\mathrm{DL} \geq 0.18$ were choosed as the filtrating parameters. A total of 14 components were choosed as effective components. The basic information of the components were shown in Table 1.

\begin{tabular}{|c|c|c|c|}
\hline Components & OB $\%$ & DL & Caco-2 \\
\hline wogonin & 30.68 & 0.23 & 0.79 \\
\hline oroxylin a & 41.37 & 0.23 & 0.76 \\
\hline mono-O-methylwightin & 103.11 & 0.4 & 0.94 \\
\hline andrographidine B_qt & 72.72 & 0.33 & 0.6 \\
\hline andrographidine C & 56.85 & 0.77 & -0.6 \\
\hline moslosooflavone & 44.09 & 0.25 & 1.01 \\
\hline andrographin & 37.57 & 0.33 & 0.73 \\
\hline andrographin F & 33.34 & 0.85 & -0.84 \\
\hline andrographidine $F \_q t$ & 77.13 & 0.45 & 0.91 \\
\hline $\begin{array}{l}\text { 14-deoxy-11-oxo- } \\
\text { andrographolide }\end{array}$ & 57.06 & 0.34 & -0.09 \\
\hline deoxyelephantopin & 105.32 & 0.4 & 0.26 \\
\hline paniculogenin & 47.66 & 0.75 & -0.02 \\
\hline 14-deoxyandrographolide & 56.3 & 0.31 & 0.17 \\
\hline $\begin{array}{c}\text { andrographolide-19- } \beta-D- \\
\text { glucoside_qt }\end{array}$ & 53.44 & 0.35 & -0.3 \\
\hline
\end{tabular}




\section{Potential targets Screen}

7242 anti-inflammatory genes were obtained from Gene Cards databases. The potential anti-inflammatory target genes of each active compound were obtained by comparing them with the anti-inflammatory genes. A total of 86 potential anti-inflammatory target genes of AP were obtained, 30 of them were shown in Table 2. The number of interacting proteins with a certain protein was shown by degree in the section 2.6. Generally, only a few nodes had high degrees occupied an important position in the whole network. The proteins in 2.6 were ranked according to the degree from high to low.

Construction andAnalysis of the Protein-Protein Interaction (PPI)

86 potential anti-inflammatory target genes were obtained. Target genes were imported to the STRING database to get the PPI network of target genes, the CSV format files were downloaded and imported to Cytoscape 3.6.1 software. (Figure 1). The PPI network was analyzed by using the network analysis tool. The genes which had a higher degree were associated with more genes. PIK3CA, IL2, JAK2, PIK3CD had a higher degree.

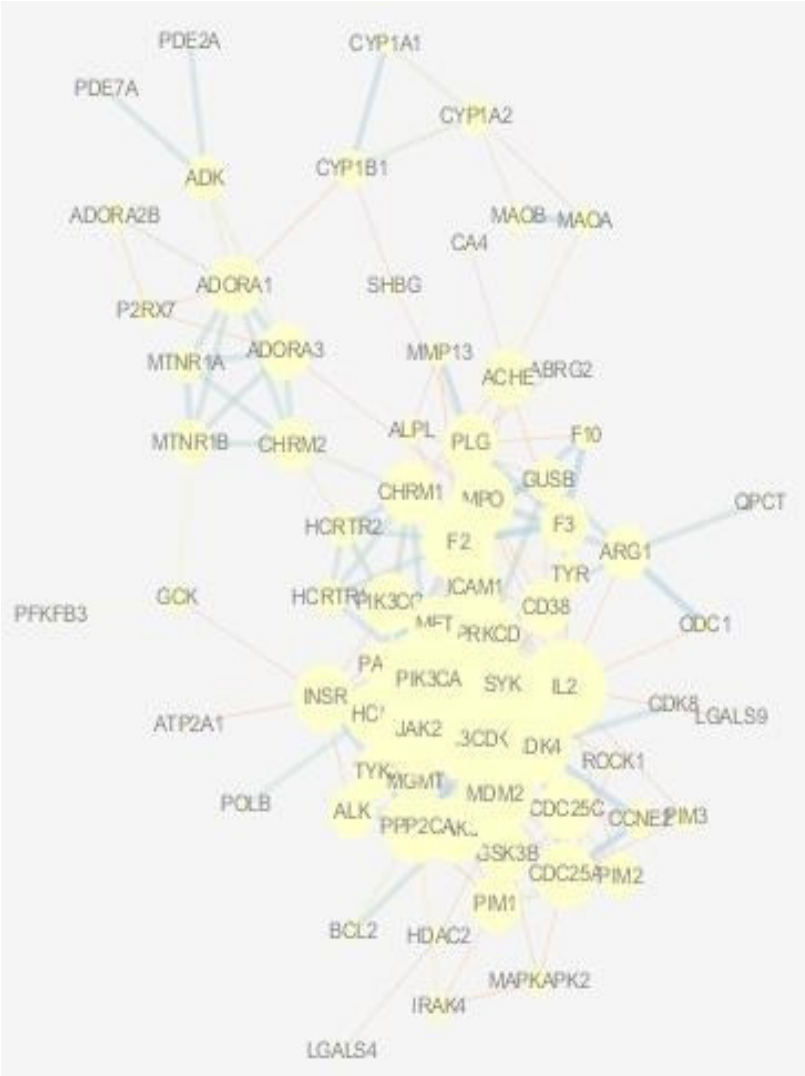

Figure 1: Protein-Protein Interaction Network obtained from STRING database and constructed by Cytoscape 3.6.1 software.

\section{Target pathway annotation and analysis}

GO and KEGG analyses were conducted on the corresponding targets of effective compounds of AP by using the DAVID database, $P<0.05$ was set. The top 10 pathways were plotted by GraphPad Prism 5 (Figure 2). KEEG pathway annotation showed that 75 potential target genes were enriched and involved 78 pathways and 65 of these pathways were significantly connected with the target genes $(P<0.05)$. The pathways had the largest number of genes involved: PI3K-Akt signaling pathway, Neuroactive ligand-receptor interaction, Measles, Viral carcinogenesis, Pathways in cancer. BP enrichment was mainly involved in the following target genes: transmembrane receptor protein tyrosine kinase signaling pathway, negative regulation of apoptotic process, peptidyl-tyrosine autophosphorylation. CC enrichment was mainly involved in the following target genes: extracellular exosome, plasma membrane, integral component of plasma membrane. MF enrichment was mainly involved in the following target genes: ATP binding, protein serine/threonine kinase activity, nonmembrane spanning protein tyrosine kinase activity.

The genes repeatedly associated with these pathways were PIK3CG, PIK3CD, PIK3CA, IL2, CYP1A1, LCK, after analysis of the 10 KEEG pathways with the strongest connection.

Construction of Component-Target-Pathway Network From the pathways that were closely related to the target genes $(P<0.05)$, the top 10 pathways with the high number of genes involved were screened, 86 target genes and 14 effective compounds were selected to establish the Component-Target-Pathway network (Figure 3). The network diagram showed the active compounds on multiple targets when AP plays anti-inflammatory effects. The degree of the effective compounds was analyzed (Table 3). The following compounds were selected for docking experiments: 14-deoxyandrographolide, paniculogenin, deoxyelephantopin, 14-deoxy-11-oxoandrographolide, rographidine F_qt.

\section{Component-Target docking}

Considering the component-target-pathway network and protein interaction network and analyzing its importance in the KEGG pathway, PIK3CG and PIK3CA were selected as the molecular docking targets.

6FH5 (PIK3CG) and 6OAC (PIK3CA) were selected from the PBD database as protein structures for docking. Pymol was used to remove ligands and solvents from the protein structures, the structures were then stored in PDB format after hydrogenation. The screened active components were compared with the positive control chemical components of the target. A minimized 

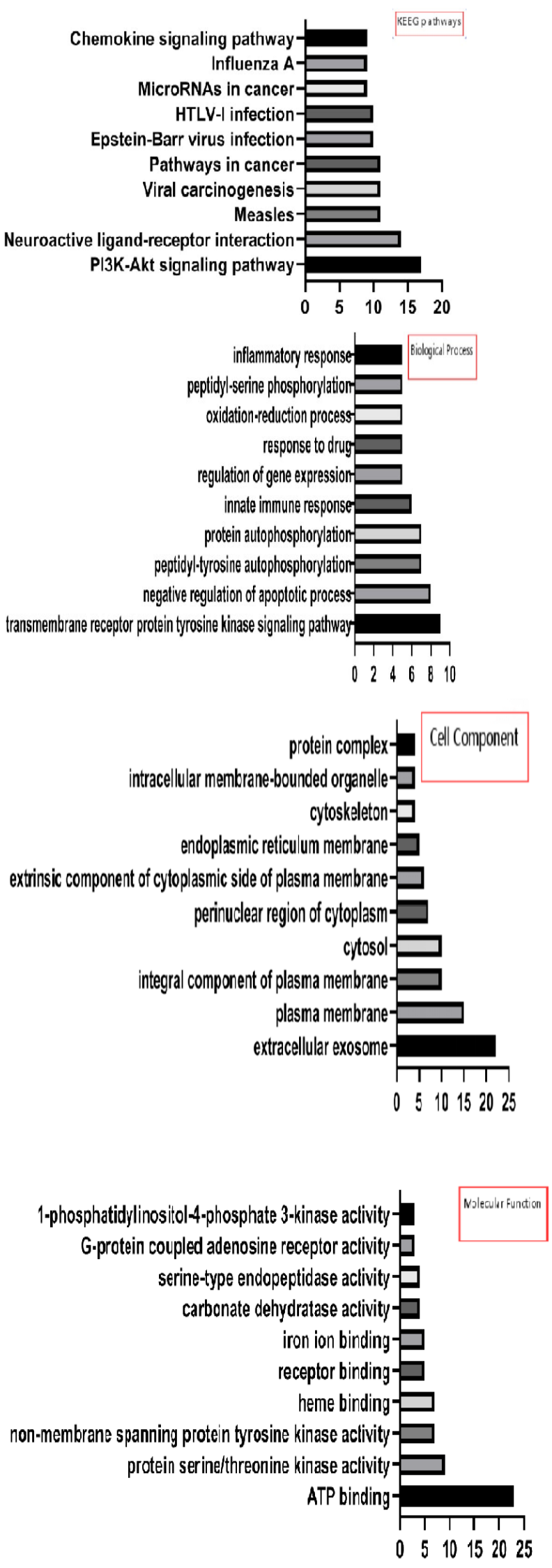

Figure 2: The top 10 components of KEEG and GO enrichment analysis. energy function was used in ChemBio3D Ultra to obtain the lowest energy conformation before storage in PDF format. The treated target protein and effective compound structures were imported to AutoDockTools 1.5.6 for molecular docking experiments. The gridbox and molecular docking parameters are shown in Table 4. After ten docking tests, the cluster with the most suitable conformation and the largest binding energy value was selected for further analysis. The largest binding energy was the binding energy between the compound and the target protein. DD $8{ }^{13}$ and $\mathrm{M} 1 \mathrm{~J}^{14}$ were respectively selected as the positive control drugs for PIK3CG and PIK3CA. The molecular docking results were shown in Table 5. When the binding energy was lower, the docking was better.

At last, we selected the PIK3CG-14deoxyandrographolide complex and PIK3CA-14deoxyandrographolide complex as the representative complexes and visualized them with Pymol. The parameters were set according to the Protein-Ligand Interaction Profiler website (https://projects.biotec. tu-dresden.de/plip-web/plip/index). The picture of the corresponding docking pattern is shown in Figure 4.

\section{DISCUSSION}

Natural have been successfully used as medicines, as well as the most important sources of drug leads, food additives, many industry relevant products for millennia. ${ }^{15}$ Modern drugs, which were developed based on single effective component, target specific biological activities and therefore are much simple to determine

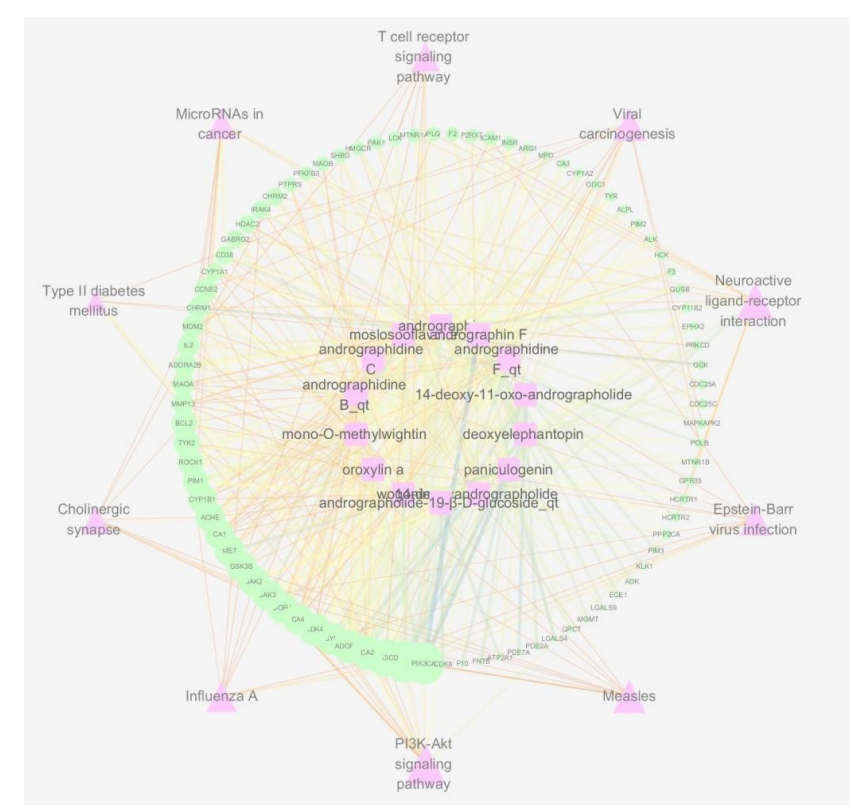

Figure 3: Component- Target-Pathway network. 
Table 2: Thirty anti-inflammation targets genes of active compounds.

\begin{tabular}{|c|c|c|c|}
\hline $\begin{array}{l}\text { Uni Prot } \\
\text { IDs }\end{array}$ & Protein name & Gene name & Degree \\
\hline P48736 & $\begin{array}{c}\text { Phosphatidylinositol 4,5-bisphosphate 3-kinase catalytic subunit } \\
\text { gamma isoform }\end{array}$ & PIK3CG & 17 \\
\hline P42336 & $\begin{array}{l}\text { Phosphatidylinositol 4,5-bisphosphate 3-kinase catalytic } \\
\text { subunit alpha isoform }\end{array}$ & PIK3CA & 17 \\
\hline O00329 & $\begin{array}{l}\text { Phosphatidylinositol 4,5-bisphosphate 3-kinase } 110 \mathrm{kDa} \\
\text { catalytic subunit delta }\end{array}$ & PIK3CD & 14 \\
\hline P30542 & Adenosine receptor $\mathrm{A} 1$ & ADORA1 & 11 \\
\hline P00918 & Carbonic anhydrase 2 & CA2 & 11 \\
\hline P11802 & Cyclin-dependent kinase 4 & CDK4 & 10 \\
\hline P43405 & Tyrosine-protein kinase SYK & SYK & 10 \\
\hline P08581 & Hepatocyte growth factor receptor & MET & 9 \\
\hline P49841 & Glycogen synthase kinase- 3 beta & GSK3B & 9 \\
\hline O60674 & Tyrosine-protein kinase JAK2 & JAK2 & 9 \\
\hline P52333 & Tyrosine-protein kinase JAK3 & JAK3 & 9 \\
\hline P0DMS8 & Adenosine receptor $\mathrm{A} 3$ & ADORA3 & 9 \\
\hline P22748 & Carbonic anhydrase 4 & CA4 & 9 \\
\hline Q16678 & Cytochrome P450 1B1 & CYP1B1 & 8 \\
\hline P22303 & Acetylcholinesterase & $\mathrm{ACHE}$ & 8 \\
\hline P00915 & Carbonic anhydrase 1 & CA1 & 8 \\
\hline Q07820 & $\begin{array}{l}\text { Induced myeloid leukemia cell differentiation protein } \\
\qquad \mathrm{Mcl}-1\end{array}$ & BCL2 & 7 \\
\hline P29597 & Non-receptor tyrosine-protein kinase TYK2 & TYK2 & 7 \\
\hline Q13464 & Rho-associated protein kinase 1 & ROCK1 & 7 \\
\hline P11309 & Serine/threonine-protein kinase pim-1 & PIM1 & 7 \\
\hline 096020 & G1/S-specific cyclin-E2 & CCNE2 & 6 \\
\hline P11229 & Muscarinic acetylcholine receptor M1 & CHRM1 & 6 \\
\hline Q00987 & E3 ubiquitin-protein ligase & MDM2 & 6 \\
\hline P60568 & Interleukin-2 & IL2 & 6 \\
\hline P29275 & Adenosine receptor $\mathrm{A} 2 \mathrm{~b}$ & ADORA2B & 6 \\
\hline P21397 & Amine oxidase & MAOA & 6 \\
\hline P45452 & Collagenase 3 & MMP13 & 6 \\
\hline P08172 & Muscarinic acetylcholine receptor M2 & CHRM2 & 5 \\
\hline Q9NWZ3 & Interleukin-1 receptor-associated kinase 4 & IRAK4 & 5 \\
\hline
\end{tabular}




\begin{tabular}{|c|c|}
\hline Chemical Name & Degree \\
\hline 14-deoxyandrographolide & 30 \\
\hline paniculogenin & 29 \\
\hline deoxyelephantopin & 28 \\
\hline 14-deoxy-11-oxo-andrographolide & 26 \\
\hline andrographidine F_qt & 26 \\
\hline andrographin $\mathrm{F}$ & 24 \\
\hline andrographin & 22 \\
\hline moslosooflavone & 21 \\
\hline andrographidine $\mathrm{C}$ & 20 \\
\hline andrographidine B_qt & 20 \\
\hline mono-O-methylwightin & 20 \\
\hline oroxylin a & 19 \\
\hline Wogonin & 18 \\
\hline andrographolide-19- $\beta$-D-glucoside_qt & 17 \\
\hline
\end{tabular}

\begin{tabular}{|c|c|c|c|c|}
\hline \multicolumn{5}{|c|}{ Table 4: Molecular docking parameters. } \\
\hline Targets & Receptors & Grid Center & Npts & Spacing \\
\hline PIK3CG & 6 FH5 & -25 & 40 & 0.375 \\
\hline & & 15 & 40 & \\
\hline & & 31 & 40 & \\
\hline PIK3CA & 6OAC & -20 & 40 & 0.375 \\
\hline & & 10 & 40 & \\
\hline & & 28 & 40 & \\
\hline
\end{tabular}

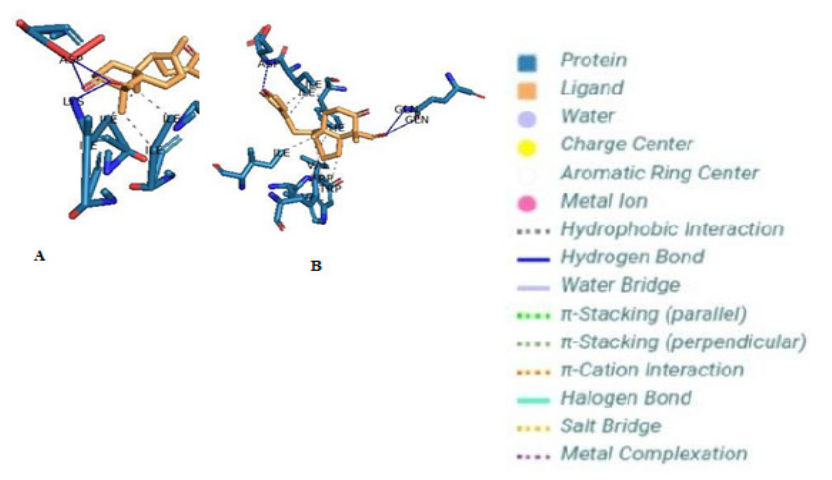

Figure 4: Docking pattern visualized by Pymol.

A. PIK3CG-14-deoxyandrographolide complex

B. PIK3CA-14-deoxyandrographolide complex
Table 5: The molecular docking results of five active compounds and two positive control drugs.

\begin{tabular}{|c|c|c|}
\hline Targets & Components & Binding Energy \\
\hline PIK3CG & 14-deoxyandrographolide & -8.19 \\
\hline & paniculogenin & -7.42 \\
\hline & deoxyelephantopin & -8.27 \\
\hline & $\begin{array}{c}\text { 14-deoxy-11-oxo- } \\
\text { andrographolide }\end{array}$ & -7.89 \\
\hline PIK3CA & $\begin{array}{c}\text { andrographidine F_qt } \\
\text { DD8(PDB Ligand code) }\end{array}$ & -7.68 \\
\hline & $\begin{array}{c}\text { 14-deoxyandrographolide } \\
\text { paniculogenin }\end{array}$ & -7.16 \\
\hline & deoxyelephantopin & -7.42 \\
\hline & $\begin{array}{c}\text { 14-deoxy-11-oxo- } \\
\text { andrographolide }\end{array}$ & -8.73 \\
\hline & $\begin{array}{c}\text { andrographidine F_qt } \\
\text { M1J(PDB Ligand code) }\end{array}$ & -7.53 \\
\hline & \begin{tabular}{l} 
and \\
\hline
\end{tabular} & -7.03 \\
\hline
\end{tabular}

the mechanism of action. ${ }^{16}$ In contrast, natural medicines such as Traditional Chinese Medicine (TCM) is made up of active compounds in the formulation that assert complex effects on human body. This "multicomponent and multi-target" nature of TCM represents a challenge in elucidating the underlying molecular mechanisms in biological systems. ${ }^{17}$ However, there are complex components in traditional Chinese medicines, the active ingredients are not clear, the targets gene of drug action or target protein is not fully identified. ${ }^{18}$ The exploration of the active ingredients and the targets need a lot of manpower, material resources, time. Network science is already making an impact on the study of complex systems and offers a promising variety of tools to understand their formation and evolution in many disparate fields from technological networks to biological systems. ${ }^{19}$

Network pharmacology describes a network analysis method to study the interrelationship between drugs, diseases, targets. It combines multidisciplinary techniques, such as systems biology, pharmacology, computer technology and it is based on the disease-gene-targetdrug interaction network. ${ }^{10}$ Network pharmacology has become a helpful tool in understanding the fine details of drug-target interactions. Using network pharmacology to investigate traditional Chinese medicine pharmacological effects and drug targets, attention should be paid to degree centrality, betweenness centrality, bridging centrality. Network-based tools for analyzing topology and especially dynamics have great potential to identify 
alternative targets for finding and developing multitarget drugs. ${ }^{20}$

ADME (drug absorption, distribution, metabolism, excretion) studies have always played a critical role in helping to optimize the pharmacokinetic (PK) properties of new drugs thereby increasing their success rate. As a consequence of the increased throughput of drug discovery, ADME studies have evolved to keep pace. These so-called "early ADME" studies, are characterized by parallel processing and higher throughput than before. ${ }^{21}$ ADME/TOX refers to the drug absorption, distribution, metabolism, excretion, toxicity. The potential power of combining computational ADME/TOX, computational biology and computational chemistry will lead to an ability to predict drug safety for a molecule much earlier and preempt synthesis of poorly scored virtual molecules. ${ }^{22}$ Final screening results show that terpenoids represents the most active ingredient, followed by flavonoids. Through a further literature search, we found that most of the previously conducted pharmacological studies on the chemical constituents of AP focused on total constituents or terpenoids constituents, but only a few of them focused on flavonoids. Previous literature reports show that andrographolide, 14-deoxy-11,12didehydroandrographolide, 14-deoxyandrographolide exhibit anti-inflammation functions. ${ }^{5,6}$ Two of the three components were successfully screened by the ADME screening method.

Cancer was linked to inflammation for the first time in the nineteenth century when in 1863, Rudolf Virchow hypothesized the functional relationship of chronic inflammation to the development of cancer. ${ }^{23}$ It has been observed that the cause of inflammation may range from microbial infections to exposure of chemicals and allergens to various pathological conditions. Inflammation is a double-edged sword having a very delicate balance between the anti- or pro-tumorigenic environments. A well-regulated acute inflammation is considered as anti-tumorigenic, while un-regulated chronic inflammation develops a pro-tumorigenic environment. ${ }^{24,25}$

KEEG pathway analysis found that the targets of AP action were enriched in addition to multiple inflammation-related pathways, PI3K-Akt signaling pathway, Neuroactive ligand-receptor interaction, Measles. In 2016, studies suggested that curcumin plays an important role in the attenuation of LPS-induced inflammatory responses in microglial cells and that the mechanisms involve down-regulation of the PI3K/Akt pathway. ${ }^{26}$ The top 3 targets were PIK3CG, PIK3CA and PIK3CD in components-target-path-ways network, they were all enriched in PI3k-Akt signaling pathway. In 2005 , it was found that pharmacological inhibition of PI3Kg can effectively ameliorate chronic inflammatory disorders such as rheumatoid arthritis. ${ }^{27}$ Meanwhile the molecular docking showed that there was a high binding between the 5 compounds and the important target proteins, as a further Verification for the subsequent network research. The anti-inflammatory activity mechanism of AP was characterized by multiple components, multiple targets and multiple pathways. The results of network pharmacology can provide certain scientific basis for subsequent experimental research. This research revealed the connection of antiinflammatory components, targets and pathways of AP and provided a new idea and method for further study on the anti-inflammatory mechanism of AP.

\section{ACKNOWLEDGEMENT}

Authors are thankful to Huanggang Normal University, College of Chemistry and Chemical Engineering for financial assistance.

\section{CONFLICT OF INTEREST}

The author(s) declared no conflicts of interest to the research, authorship and/or publication of this article.

\section{ABBREVIATIONS}

AP: Andrographis paniculata (Burm. f.) Wall. ex Nees; ADME: drug absorption, distribution, metabolism, excretion; TCM: Traditional Chinese Medicine.;

\section{REFERENCES}

1. Hossain MS, Urbi Z, Sule A, Rahman KMH. Andrographis paniculata (Burm. f.) Wall. ex Nees: A Review of Ethnobotany, Phytochemistry, and Pharmacology. The Scientific World Journal. 2014;1-28.

2. Parichatikanond W, Suthisisang C, Dhepakson P, Herunsalee A. Study of anti-inflammatory activities of the pure compounds from Andrographis paniculata (Burm. f.) Nees and their effects on gene expression. International Immunopharmacology. 2010;10(11):1361-73.2.

3. Dai Y, et al. Overview of pharmacological activities of Andrographis paniculata and its major compound andrographolide. Critical Reviews in Food Science and Nutrition. 2018.

4. Chao WW, Lin BF. Isolation and identification of bioactive compounds in Andrographis paniculata (Chuanxinlian). Chinese medicine. 2010;5(1):1-5.

5. Dai G-F, Zhao J, Jiang Z-W, Zhu L-P, Xu H-W, Ma W-Y, et al. Anti-inflammatory effect of novel andrographolide derivatives through inhibition of NO and PGE2 production. International Immunopharmacology. 2011;11(12):2144-9.

6. Shaikh RU, Meshram RJ, Gacche RN. An investigation on in vitro antiinflammatory and antiproliferative potential of isolated Labdane diterpenoids from Andrographis paniculata (Burm. f.) wall. Ex nees: An important medicinal plant prescribed in Ayurveda. European Journal of Integrative Medicine. 2019;32:100983.

7. Zou W, Xiao Z, Wen X, Luo J, Chen S, Cheng Z, et al. The anti-inflammatory effect of Andrographis paniculata (Burm. f.) Nees on pelvic inflammatory 
disease in rats through down-regulation of the NF-KB pathway. BMC Complementary and Alternative Medicine. 2016;16(1):1-7.

8. Bera R, Ahmed SK, Sarkar L, Sen T, Karmakar S. Pharmacokinetic analysis and tissue distribution of andrographolide in rat by a validated LC-MS/MS method. Pharmaceutical Biology. 2014;52(3):321-9.

9. Panossian A, et al. Pharmacokinetic and oral bioavailability of andrographolide from Andrographis paniculata fixed combination Kan Jang in rats and human. Phytomedicine. 2000;7(1):351-364.

10. Hopkins AL. Network pharmacology: The next paradigm in drug discovery. Nature Chemical Biology. 2008;4(11):682-90.

11. Xiao W, Yang Y, Wu H, Xiong Y. Predicting the Mechanism of the Analgesic Property of Yanhusuo Based on Network Pharmacology. Natural Product Communications. 2019;14(10):1934578X19883071.

12. Xiao Z, Morrisnatschke SL, Lee KH. Strategies for the Optimization of Natural Leads to Anticancer Drugs or Drug Candidates. Medicinal Research Reviews. 2016;36(1) :32-91.

13. Barlaam B, et al. Discovery of a series of 3-cinnoline carboxamides as orally bioavailable, highly potent and selective ATM inhibitors. ACS Medicinal Chemistry Letters. 2018;9(8): 809-814.

14. Rageot D, et al. (S)-4-(Difluoromethyl)-5-(4-(3-methylmorpholino)-6morpholino-1, 3, 5-triazin-2-yl) pyridin-2-amine (PQR530), a Potent, Orally Bioavailable and Brain-Penetrable Dual Inhibitor of Class I PI3K and mTOR Kinase. Journal of Medicinal Chemistry. 2019;62(13): 6241-6261.

15. Li F, Wang Y, Li D, Chen Y, Dou QP. Are we seeing a resurgence in the use of natural products for new drug discovery?. Expert Opinion on Drug Discovery. 2019;14(5):417-20.15.

16. Knowles J, Gromo G. Target selection in drug discovery. Nature Reviews Drug Discovery. 2003;2(1):63-69.
17. Kim HU, Ryu JY, Lee JO, Lee SY. A systems approach to traditional oriental medicine. Nature Biotechnology. 2015;33(3): 264-8.

18. Zhang R, Yu S, Bai H, Ning K. TCM-Mesh: The database and analytical system for network pharmacology analysis for TCM preparations. Scientific Reports. 2017;7(6):2821.

19. Nacher JC, Schwartz J. A global view of drug-therapy interactions. BMC Pharmacology. 2008;8(5):5

20. Zhang GB, Li QY, Chen QL, Su SB. Network Pharmacology: A New Approach for Chinese Herbal Medicine Research. Evidence-Based Complementary and Alternative Medicine. 2013;1-9.

21. Thompson TN. Early ADME in Support of Drug Discovery: The Role of Metabolic Stability Studies. Current Drug Metabolism. 2000;1(5):215-41

22. Durán-Iturbide NA, Díaz-Eufracio BI, Medina-Franco JL. In silico ADME/ Tox Profiling of Natural Products: A Focus on BIOFACQUIM. ACS Omega. 2020;5(26):16076-84.

23. Balkwill FR, Mantovani A. Inflammation and cancer: back to Virchow?. The Lancet. 2001;357(9255):539-45

24. Coussens LM, Werb Z. Inflammation and cancer. Nature. 2002;420(5):860-7.

25. Mantovani A, Allavena P, Sica A, Balkwill FR. Cancer-related inflammation. Nature. 2008;454(1):436-44.

26. Cianciulli A, Calvello R, Porro C, Trotta T, Salvatore R, Panaro MA. PI3k/ Akt signalling pathway plays a crucial role in the anti-inflammatory effects of curcumin in LPS-activated microglia. International Immunopharmacology. 2016;36:282-90.

27. Camps M, Rückle T, Ji H, Ardissone V, Rintelen F, Shaw J, et al. Blockade of $\mathrm{PI} 3 \mathrm{~K} Y$ suppresses joint inflammation and damage in mouse models of rheumatoid arthritis. Nature Medicine. 2005;11(9):936-43.

\section{SUMMARY}

The combination of PIK3CG and 14-deoxyandrographolide were simulated by AutoDock1.5.6.

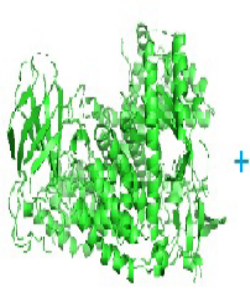

PIK3CG

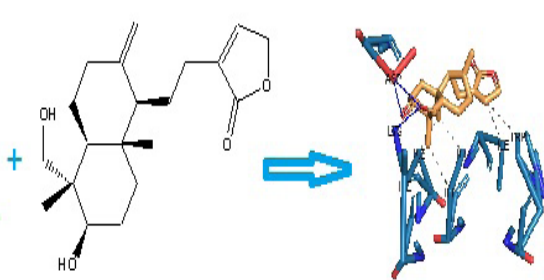

14-deoxyandrographolide

\section{About Authors}

Wenping Xiao, female, master, born in February 1980, graduated from Hubei University of Traditional Chinese Medicine, research direction is extraction and separation of natural medicines. She is mainly engaged in the research of natural pharmaceutical chemistry. She can independently complete the extraction, separation and purification of active compounds in natural medicines, and analyze the structure of compounds by NMR, IR, MS, and can use modern methods such as HPLC for quantitative analysis. At the same time, she also have a lot of research on the information collection of pharmaceutical research.

Cite this article: Xiao Wp, Zhang Wj, Xiong Xj, Xu Ly. Network Pharmacology-based on the Active Components and Molecular Mechanisms of Andrographis paniculata (Burm. f.) Wall. ex Nees in Treating Inflammation. Indian $\mathrm{J}$ of Pharmaceutical Education and Research. 2021;55(3):765-73. 